\title{
An unusual but reversible cause of ventricular fibrillation
}

\author{
${ }^{1} \mathrm{I}$ Merinopoulos, ${ }^{2} \mathrm{~V}$ Vassiliou, ${ }^{3} \mathrm{JN}$ Porter, ${ }^{4} \mathrm{~S}$ Acton, ${ }^{5} \mathrm{DR}$ Braganza \\ ${ }^{1}$ Cardiology Senior House Officer, Papworth Hospital, Cambridge, UK; ${ }^{2}$ Cardiology SpR, Papworth Hospital, Cambridge, UK; ${ }^{3}$ Cardiology \\ Consultant, Peterborough City Hospital, Peterborough, UK; ${ }^{4}$ Renal Consultant, Peterborough City Hospital, Peterborough, UK; ${ }^{5}$ Cardiology \\ Consultant, Papworth Hospital, Cambridge, UK and Peterborough City Hospital, Peterborough, UK
}

\begin{abstract}
A 6I-year-old woman was admitted with general malaise, chest pain and breathlessness. During her inpatient stay she sustained a ventricular fibrillation (VF) arrest which was successfully terminated with direct current cardioversion. Cardiac investigations revealed poor left ventricular systolic function but unequivocally normal coronary arteries. During the course of her admission a macular rash developed and following investigations including a renal biopsy, a new diagnosis of systemic lupus erythematosus (SLE) and related myocarditis was reached. First presentation of lupus with myocarditis and VF is uncommon, however reaching the correct diagnosis is important as due to the reversible nature of the condition and improvement in left ventricular systolic function with medical therapy, an implantable cardioverter defibrillator (ICD) might not be appropriate. Our case report demonstrates the importance of screening for reversible conditions when considering ICD therapy for secondary prevention of malignant arrhythmias.
\end{abstract} Correspondence to I Merinopoulos 9 Warren Chase, Ipswich IP5 2WZ, UK

tel. +44 (0)780 060 I57I e-mail

i_merinopoulos@hotmail.com

KEYWORDS Lupus, SLE, myocarditis, ventricular fibrillation, cardiac arrest

DECLARATION OF INTERESTS No conflicts of interest declared.

\section{INTRODUCTION}

Survivors of cardiac arrest constitute a challenging group of patients to treat; they require extensive investigations to identify the cause and determine the most appropriate management. If the pathology leading to arrhythmias cannot be treated, many patients will benefit from an implantable cardioverter defibrillator (ICD) to prevent future arrhythmias. However, in some patients the arrhythmia could relate to a reversible pathology. For these patients, treating the underlying pathology is therefore more important and safer than implanting an ICD. According to the American College of Cardiology/ American Heart Association/the Heart Rhythm Society (ACC/AHA/HRS) 2008 guidelines, ICD is indicated only after the exclusion of any completely reversible causes.' This case demonstrates a rare, yet completely reversible pathology leading to ventricular fibrillation (VF).

\section{CASE REPORT}

A 61-year-old healthy woman presented with a threemonth history of general deterioration, chest pain and shortness of breath on exertion. She also complained of left-sided abdominal pain, loss of appetite and a $12 \mathrm{~kg}$ weight loss. Her symptoms appeared to have started after a wisdom tooth extraction which had been complicated by infection and treated with metronidazole. As she had microcytic anaemia, gastrointestinal symptoms and weight loss, her GP had organised an oesophagogastroduodenoscopy which revealed chronic gastritis and omeprazole was initiated.

Her past medical and family history were otherwise unremarkable. She was a non-smoker and did not drink alcohol. There were no known drug allergies and her only regular prescribed or recreational medication was omeprazole.

Shortly after admission, she became tachycardic (heart rate $[H R]=140$, sinus tachycardia), tachypnoeic (respiratory rate $[R R]=30$ ), febrile (temperature $=38.5$ ${ }^{\circ} \mathrm{C}$ ) and hypoxic requiring 15 litres of oxygen via a nonrebreather mask to maintain saturation above $95 \%$. On respiratory auscultation she was found to have bibasal crackles and decreased air entry at the left base; on abdominal examination she had tenderness in the left side of the abdomen. Heart sounds were normal without any murmurs, the jugular venous pressure was not elevated and there was no peripheral oedema. Blood pressure was maintained at $150 / 90 \mathrm{mmHg}$ and she required a short admission to the intensive therapy unit (ITU) for respiratory support. Initial investigations revealed microcytic anaemia (haemoglobin $[\mathrm{Hb}]=98 \mathrm{~g} / \mathrm{L}$, mean corpuscular volume $[\mathrm{MCV}]=77.8$ ), lymphopaenia $(\mathrm{Lymph}=|x| 09 / \mathrm{L})$, raised inflammatory markers (C-reactive protein [CRP] $=27$ ), acute kidney injury (urea $[\mathrm{Ur}]=12.9 \mathrm{mmol} / \mathrm{L}$, creatinine $[\mathrm{Cr}]=153 \mathrm{umol} / \mathrm{L}$, 
potassium $[K]=4.9 \mathrm{mmol} / \mathrm{L})$, hypoalbuminaemia $(\mathrm{Alb}=27$ $\mathrm{g} / \mathrm{L}$ ) and raised troponin (Trop $=54 \mathrm{ng} / \mathrm{L}$, normal $<27 \mathrm{ng} / \mathrm{L}$ ). Her thyroid function tests were normal (thyroid stimulating hormone $[\mathrm{TSH}]=\mathrm{I}$.2). She also had proteinuria and microscopic haematuria. The electrocardiogram (ECG) showed first degree heart block with left axis deviation and non-specific T-wave inversion with a normal QT interval (Figure 1). Chest radiography revealed cardiomegaly, pulmonary congestion and a large left pleural effusion which was subsequently aspirated and found to have numerous macrophages without any atypical cells or evidence of malignancy. The initial differential diagnosis included chest sepsis, subacute bacterial endocarditis, abdominal sepsis or other intraabdominal pathology and following discussion and advice from the microbiologists the patient was started on intravenous broad spectrum antibiotics with benzylpenicillin and gentamicin.

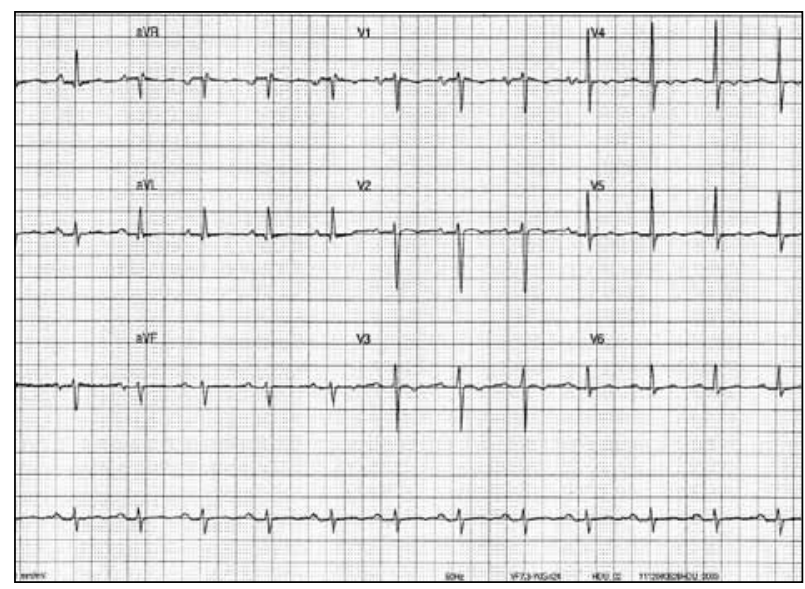

FIGURE I Electrocardiogram (ECG) showing first degree heart block, left axis deviation and non-specific T-wave inversion with normal QT interval.

Further investigation with a computed tomography (CT) scan, a pulmonary angiography and a CT of the abdomen/ pelvis demonstrated cardiomegaly with prominent pericardial effusion and congested lungs but no pulmonary emboli or significant abdominal abnormalities. These findings raised the possibility of cardiac failure secondary to a viral cardiomyopathy or infective endocarditis with pulmonary congestion. Echocardiography showed severely impaired left ventricular systolic function with mild mitral regurgitation and a moderate sized global pericardial effusion with no evidence of tamponade physiology (Figure 2).

Two days after admission the patient sustained a VF arrest, which was successfully treated with a single direct current shock. At the time of cardiac arrest her renal function and electrolytes were stable $(\mathrm{K}=4.5 \mathrm{mmol} / \mathrm{L}, \mathrm{Ur}=12.8 \mathrm{mmol} / \mathrm{L}$, $\mathrm{Cr}=167 \mathrm{umol} / \mathrm{L}, \mathrm{Mg}=0.66 \mathrm{mmol} / \mathrm{L})$ and the post-arrest ECG had a normal QTc without any significant or specific abnormalities. She was immediately treated with

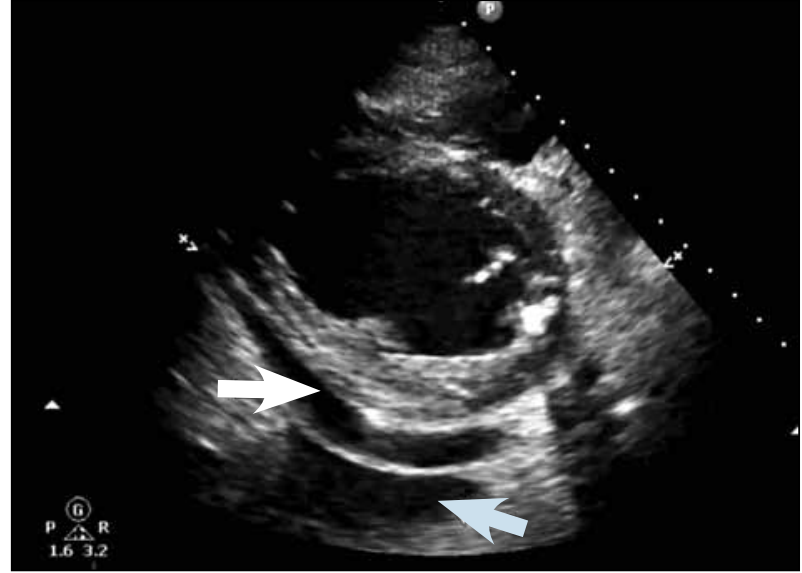

FIGURE 2 Short axis parasternal view showing the pericardial (white arrow) and pleural effusions (blue arrow).

intravenous amiodarone ( $300 \mathrm{mg}$ over one hour followed by $900 \mathrm{mg}$ over 23 hours) and transferred to the regional cardiothoracic centre for urgent inpatient cardiac investigations. Coronary angiography demonstrated unequivocally smooth unobstructed epicardial vessels with no evidence of angiitis (Figure 3), and echocardiography confirmed poor systolic function with an ejection fraction (EF) estimated at $25 \%$.

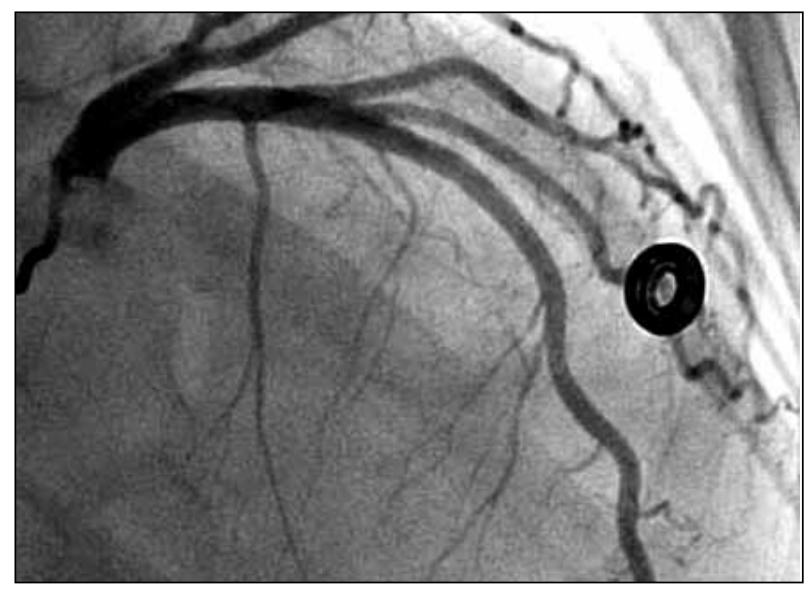

FIGURE 3 Cardiac catheterisation showing unobstructed left coronary system arteries.

Two days later she developed an itchy, erythematous, macular rash on her lower back and abdomen which subsequently showed up as a 'butterfly' distribution on her face. We investigated for systemic lupus erythematosus (SLE) and blood tests for anti-nuclear/ double-stranded DNA/extractable nuclear antigen/antiRo (ANA/dsDNA/ENA/anti-Ro) antibodies were positive. The dsDNA results were strongly positive at a value of 191 (normal range 0-10). An ICD was considered, but given the potential reversibility of left ventricular systolic function with immunosuppression in the context of lupus myocarditis, it was felt appropriate and safe to defer insertion until the patient's response to medical therapy had been fully assessed. 
With a working diagnosis of VF secondary to acute SLE myocarditis, the patient underwent a renal biopsy, which revealed immune complex-mediated mesangioproliferative glomerulonephritis compatible with class IIA or III lupus. Following the results of the renal biopsy the patient was initiated on prednisolone and mycophenolate mofetil (MMF). Both her symptoms and left ventricular systolic function started to improve soon after. She was allowed home on oral medications including prednisolone, MMF, aspirin, bisoprolol, frusemide and amiodarone. An angiotensin-converting-enzyme (ACE) inhibitor was not initiated due to renal impairment.

During the outpatient visit two months later, both a magnetic resonance imaging (MRI) scan and a transthoracic echocardiography confirmed significant improvement of the LV systolic function with an EF of 45-50\% (Figure 4). At the time of the MRI, renal function, although improving, did not allow safe use of gadolinium. Given the improvement in both LV function and the patient's symptoms, the diuretic and amiodarone were discontinued. It was also concluded that with a lownormal LV function she no longer had an indication for an ICD.When last reviewed 18 months after the event she remained well. Her only medications were prednisolone 5 $\mathrm{mg}$ once daily and MMF $750 \mathrm{mg}$ twice daily (dose reduced from I g twice daily due to gastrointestinal side-effects) and her EF had normalised further to $55 \%$.

\section{DISCUSSION}

Systemic lupus erythematosus is a classical multi-system autoimmune disease often described as 'the disease with 1,000 faces' in view of the variety of clinical presentations and organs that it can affect. The heart is involved in more than $50 \%$ of patients and every cardiac structure can be affected: pericardium, myocardium, endocardium, coronary vessels and the conduction system. ${ }^{2}$ Clinically overt myocarditis is considered an uncommon complication of lupus and it is not included in the standard diagnostic criteria for SLE. ${ }^{3}$

\section{REFERENCES}

I Epstein AE, DiMarco JP, Ellenbogen KA et al.ACC/AHA/HRS 2008 guidelines for device-based therapy of cardiac rhythm abnormalities: executive summary. Circulation 2008; I 17:2820-40. http://dx.doi. org/I0.II6I/CIRCUALTIONAHA.108.18974I

2 Doria A, laccarino L, Sarzi-Puttini $P$ et al. Cardiac involvement in systemic lupus erythematosus. Lupus 2005; | 4:683-6. http://dx.doi. org/10.1191/0961203305lu2200oa

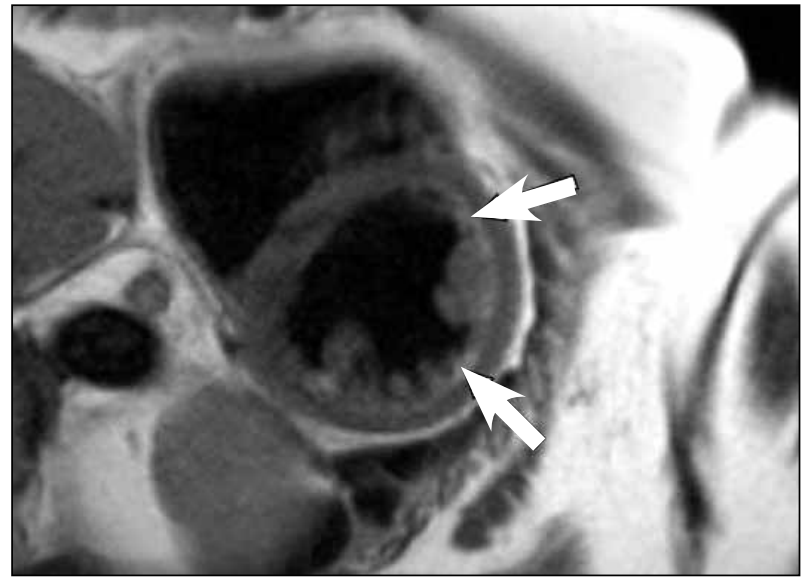

FIGURE 4 Focus of high signal (arrows) suggesting presence of oedema in left ventricular wall. On cine images the ejection fraction (EF) was estimated at $45-50 \%$.

There have been very few case reports where acute myocarditis was the initial clinical presentation of SLE. ${ }^{2}$ Our case demonstrates a new diagnosis of SLE that manifested itself as lupus myocarditis complicated with heart failure and VF arrest. Given the high rate of atherosclerosis in patients with SLE even at a young age, it is reasonable to consider that a VF arrest in the context of lupus has an ischaemic aetiology and should therefore be initially investigated with a coronary angiography. ${ }^{4}$ However, if the coronary vessels are normal, as in our patient, or show non-obstructive disease, then it is imperative to recognise that lupus myocarditis complicated with VF arrest can be the initial presentation of SLE. This is particularly important as lupus myocarditis can be a potentially reversible condition with a short duration if diagnosed and treated appropriately (as in our case). According to the ACC/ AHA/HRS 2008 guidelines, 'ICD therapy is indicated in survivors of cardiac arrest due to ventricular fibrillation after evaluation to define the cause of the event and to exclude any completely reversible causes'.' The decision to employ ICD therapy in survivors of VF arrest should therefore include a careful search for any treatable and reversible causes, as a minority of patients might not benefit from such a device.

3 Wijetunga M, Rockson S. Myocarditis in systemic lupus erythematosus Am J Med 2002; II3:419-23. http://dx.doi.org//0.1016/S00029343(02)0I223-8

4 Ashrafi R, Garg P, McKay E et al.Aggressive cardiac involvement in systemic lupus erythematosus: a case report and a comprehensive literature review. Cardiol Res Pract 20 I I; 20 I I:578390. 